\title{
CO oxidation over ZnO films on Pt(111) at near-atmospheric pressures
}

\author{
Y. Martynova ${ }^{1}$, B.-H. Liu' ${ }^{1}$, M.E. McBriarty ${ }^{1,2}$, I.M.N. Groot ${ }^{1}$, M.J. Bedzyk ${ }^{2}$, S. Shaikhutdinov ${ }^{1 *}$, \\ H.-J. Freund ${ }^{1}$ \\ ${ }^{1}$ Abteilung Chemische Physik, Fritz-Haber-Institut der Max-Planck-Gesellschaft \\ ${ }^{2}$ Department of Materials Science and Engineering, Northwestern University
}

\begin{abstract}
Well-ordered ultrathin $\mathrm{ZnO}(0001)$ films were grown on $\mathrm{Pt}(111)$ in a layer-by-layer mode. The reactivity of the films as a function of the film thickness and coverage was examined by the $\mathrm{CO}$ oxidation reaction at near-atmospheric pressures. At low temperatures $(\sim 450 \mathrm{~K}), \mathrm{CO}_{2}$ production is found to be much higher on the films of partial coverage than on dense $\mathrm{ZnO}(0001)$ films and bare $\mathrm{Pt}(111)$. Under reaction conditions, monolayer islands and an entire monolayer film transform into two-monolayers thick islands, which dominate the surface of the active catalysts. The results provide an adequate structural model for elucidating the reaction mechanism on the oxide/metal boundary at technologically relevant conditions.
\end{abstract}




\section{Introduction}

Thin oxide films grown on metal single crystals are suitable supports for modeling heterogeneous catalysts. Recent studies demonstrated that ultra-thin films, those with thickness of only a few monolayers, may exhibit interesting catalytic properties which are different from those of thicker films or their bulk counterparts. $(1 ; 2)$ For example, an iron oxide $\mathrm{FeO}(111)$ monolayer (ML) film grown on $\mathrm{Pt}(111)$ shows a higher $\mathrm{CO}$ oxidation rate than multinanometer thick iron oxide films or clean Pt(111), when the reaction takes place at nearatmospheric pressures and low temperatures ( $450 \mathrm{~K}$ ).(3) Accordingly, the encapsulation of Pt nanoparticles by an FeO layer, which occurs as a result of strong metal-support interaction between $\mathrm{Pt}$ and $\mathrm{Fe}_{3} \mathrm{O}_{4}(111)$, resulted in higher activity as compared to pure Pt particles.(4) A higher reaction rate than on $\mathrm{Pt}(111)$ has been predicted for a $2 \mathrm{ML}$-thick $\mathrm{MgO}(100)$ film on $\mathrm{Ag}(100) .(5)$ Although CO oxidation follows different reaction mechanisms for these two systems, the important steps include charge transfer from the oxide/metal interface to oxygen which is accompanied by an oxide lattice distortion. It is therefore anticipated that the film thickness may affect the reaction, particularly when charge transfer is the rate limiting step. If the film thickness does matter, then this allows one to tune the reactivity and selectivity of such an "inverted" catalyst, where an oxide phase is supported by a metal, in contrast to a traditional oxide-supported metal catalyst.

In fact, such ideas have already been put forward, most notably by Vol'kenstein(6) and Schwab(7) (see also a critical review by Slinkin et al. (8)), who developed the so-called "electron theory of catalysis" based, in essence, on the concept of Schottky barriers. However, in that time the preparation and atomic scale characterization of thin oxide films was not feasible. Establishing direct relationships between the film thickness and the reactivity requires controllable growth of well-ordered oxide films in a layer-by-layer mode. Furthermore, in order to elucidate the role of the oxide phase in the reaction, it is necessary to compare the reactivity of various metal oxide films on the same support.

Bearing all this in mind and having the above-mentioned $\mathrm{FeO}(111) / \mathrm{Pt}(111)$ system as a benchmark, we addressed here the preparation and reactivity of zinc oxide films on $\mathrm{Pt}(111)$. 
Note that bulk ZnO has the wurtzite structure which is, when projected to the (0001) plane, somewhat similar to $\mathrm{FeO}(111)$. Both structures consist of alternating close-packed layers of oxygen and metal ions. The growth of well-ordered $\mathrm{ZnO}$ films was previously reported in the literature using $\mathrm{Ag}(111)$ and $\mathrm{Pd}(111)$ single crystal substrates. With the help of surface $\mathrm{x}$-ray diffraction and scanning tunneling microscopy (STM), Tusche et al.(9) reported that 2 to $4 \mathrm{ML}$ thick $\mathrm{ZnO}(0001)$ films grown on $\mathrm{Ag}(111)$ are somewhat depolarized. That is the surface-normal displacement of $\mathrm{Zn}$ and $\mathrm{O}$ atoms is reduced such that the film resembles the co-planar sheets in the hexagonal boron nitride (or graphite) structure. The transition to the bulk wurtzite structure is accompanied by considerable surface roughening. On $\mathrm{Pd}(111)$, Weirum et al.(10) also observed layer-by-layer growth for ZnO films up to at least $5 \mathrm{ML}$. Low energy electron diffraction (LEED) and STM results together with density functional theory calculations suggested that the graphite-like structure is thermodynamically the most stable phase over a large range of oxygen chemical potentials, before it converges to the bulk-type wurtzite structure at a film thickness above $4 \mathrm{ML}$.

Numerous experimental and theoretical studies of $\mathrm{ZnO}$ single crystal surfaces, recently reviewed by Wöll,(11) were primarily focused on the polar stability and adsorption properties of $\mathrm{ZnO}$ surfaces with respect to methanol synthesis and related reactions which occur on $\mathrm{ZnO}$ based catalysts. $\mathrm{CO}$ oxidation has also been studied on $\mathrm{ZnO}$ powders and films ((12-15) and references therein). Early results revealed (see survey in ref.(15)) that the activity of $\mathrm{ZnO}$ surfaces strongly depends on the temperature, doping, illumination, and sample preparation. A comparative study on the reactivity of polycrystalline and single crystal ZnO in CO oxidation by Göpel and co-workers(15) showed that the elementary steps of the catalytic oxidation of CO on both systems can be explained quantitatively on the basis of electronic charge transfer reactions accompanied by band bending. Consequently, it was suggested that the catalytic activity can be enhanced significantly by applying an electric field. These authors also concluded that $\mathrm{CO}$ oxidation is a face-specific reaction which occurs only at the non-polar $\mathrm{ZnO}(10-10)$ surface.

Weiss and Folman(12) studied CO oxidation activity over ZnO films fabricated on Ag foil by radio-frequency sputtering as a function of the film thickness. The authors found that the 
elementary constants in the rate equations for $\mathrm{CO}$ oxidation depend on the film thickness. Good agreement between experimental results and theory based on the Schottky model together with the rigid band model has been observed. Note, however, that the thinnest films studied in this work were about $20 \mathrm{~nm}$ in thickness, and the reaction was carried out at elevated temperatures, i.e. 600-650 K.

To continue our previous works on CO oxidation over ultrathin oxide films, we studied here the reactivity of $\mathrm{ZnO}$ films on $\mathrm{Pt}(111)$ at low temperatures ( $\sim 50 \mathrm{~K})$ and near-atmospheric pressures. The films were characterized by LEED, Auger electron spectroscopy (AES), temperature programmed desorption (TPD) and STM. The structure-reactivity relationships suggest the oxide/metal boundary as the most active region under the conditions studied.

\section{Experimental}

The experiments were performed in two ultrahigh vacuum (UHV) chambers (base pressure below $2 \times 10^{-10}$ mbar) equipped with LEED, AES (both from Specs), and quadrupole mass-spectrometers (QMS, from Hiden) for TPD measurements. One chamber houses a goldplated high-pressure reaction cell for reactivity studies at atmospheric pressures using a gas chromatograph (GC, from Agilent) for the gas composition analysis. The double-side polished $\mathrm{Pt}(111)$ crystal was spot-welded to thin Ta wires for resistive heating. The second chamber is equipped with a STM (Omicron) and has an additional Au-plated cell, separated from the main chamber by the gate-valve, for high-pressure treatments to mimic technologically relevant reaction conditions. The Pt(111) crystal is clamped to an Omicron sample holder. In both setups, the sample temperature was measured by a Type $\mathrm{K}$ thermocouple spot-welded to the edge of the crystal.

A clean Pt(111) surface was obtained by cycles of $\mathrm{Ar}^{+}$-sputtering and annealing in UHV and $\mathrm{O}_{2}$. Zinc was deposited by heating a $\mathrm{Zn}$ rod ( $1 \mathrm{~mm}$ in diameter, $99.99 \%$, Goodfellow) to $480-$ $520 \mathrm{~K}$ by passing current through a tungsten wire wrapped around the rod. The $\mathrm{Zn}$ source is shielded by a metal cylinder having a small orifice ( $~ 5 \mathrm{~mm}$ in diameter) and placed about $2 \mathrm{~cm}$ away from a crystal. The deposition flux was controlled via a Type $\mathrm{K}$ thermocouple spot-welded 
to the edge of the $\mathrm{Zn}$ rod. The zinc oxide films were prepared by $\mathrm{Zn}$ deposition onto clean $\mathrm{Pt}(111)$ in $10^{-7}$ mbar $\mathrm{O}_{2}$ followed by oxidation at $600 \mathrm{~K}$ in $10^{-6}-10^{-5} \mathrm{mbar}_{2}$ for $20 \mathrm{~min}$. For reactivity studies, oxide films were grown on both sides of the crystal.

The reaction gas mixture, consisting of $10 \mathrm{mbar} \mathrm{CO}$ and $50 \mathrm{mbar}_{2}$ balanced with He to 1 bar, was dosed at room temperature and circulated with a velocity of about $3 \mathrm{ml} \mathrm{min}^{-1}$ for ca. $20 \mathrm{~min}$. Then the sample was heated to $450 \mathrm{~K}$ with a rate of $1 \mathrm{Ks}^{-1}$, and the reaction was monitored by GC.

STM images shown herein were taken with Pt-Ir tips at bias voltages of $1-2 \mathrm{~V}$ and tunneling currents of $0.5-0.7 \mathrm{nA}$. The images were subjected to a plane correction.

\section{Results and discussion}

Figure 1 shows LEED patterns and Auger spectra of the "as-prepared" films with increasing thickness. The surface coverage was measured by CO titration of Pt sites using TPD of CO that desorbs from Pt(111) at $300-500 \mathrm{~K}$ (see Fig. S1). For more than one monolayer coverage, the nominal thickness was measured by integrating the $32 \mathrm{amu}\left(\mathrm{O}_{2}\right)$ desorption signal during film decomposition at T >950 K (Fig. S2). In addition, the thickness was monitored by AES via attenuation of the Pt-related signals (Fig. S3).

The diffraction spots of $\mathrm{ZnO}(0001)-(1 \times 1)$ are aligned with those of $\mathrm{Pt}(111)$ and show up together with the surrounding hexagonal spots. The latter can be straightforwardly assigned to a coincident $\mathrm{Pt}(111)-(6 \times 6)$ superstructure, similar to that observed on $\mathrm{ZnO} / \mathrm{Pd}(111),(10)$ that arises due to the mismatch between the lattice constants of $\mathrm{ZnO}(0001)$ and $\mathrm{Pt}(111)$ (3.25 and $2.78 \AA$, respectively). Basically, six Pt(111) surface unit cells coincide with five $\mathrm{ZnO}(0001)$ cells along the surface lattice directions. The surface $\mathrm{ZnO}$ lattice is strained by about $3 \%$ versus the bulk lattice. Above 2-3 ML, the films only show a relatively diffuse pattern of $\mathrm{ZnO}(0001)$ with a high background intensity. The ZnO-related spots were sometimes streaky in the "tangential" direction, thus indicating the presence of domains slightly misaligned with respect to the metal support underneath. 

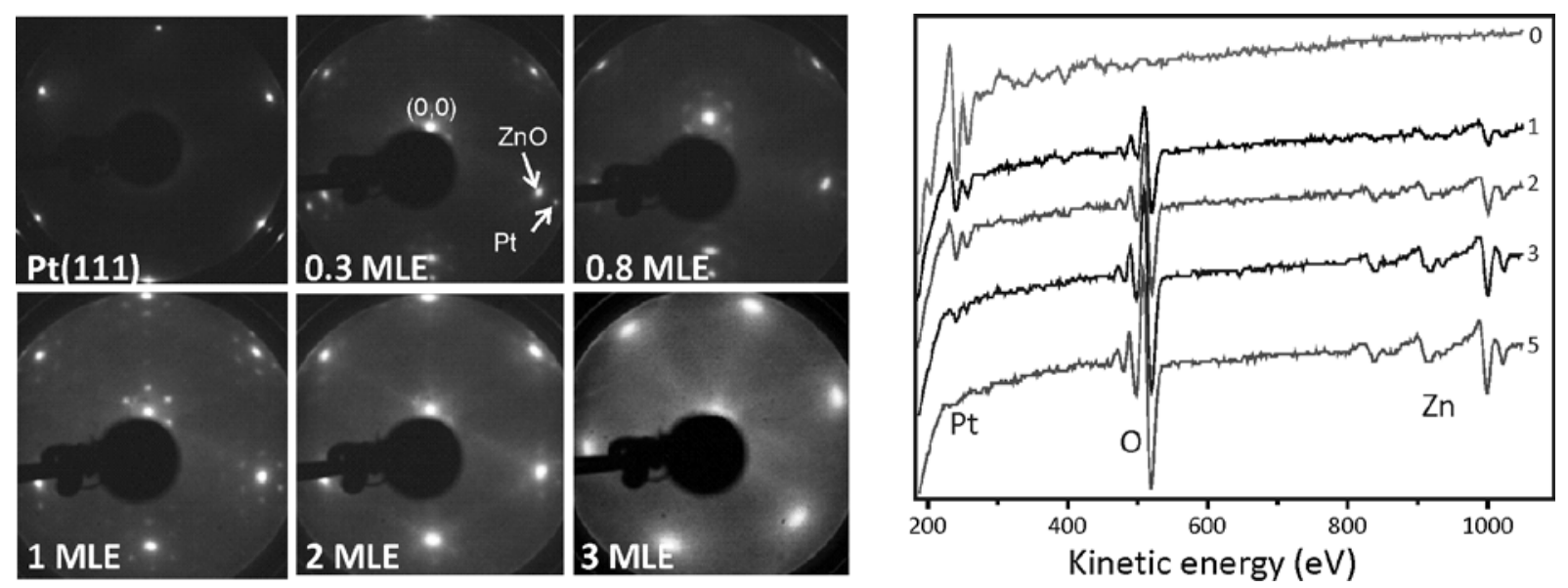

Figure 1. LEED patterns (at $60 \mathrm{eV}$, left panel) and Auger spectra (right panel) of $\mathrm{ZnO}$ films grown on $\mathrm{Pt}(111)$ at the nominal film thicknesses as indicated. Monolayer equivalent (MLE) values are shown to the right of the Auger spectra.

To address the morphology of the ZnO films we performed STM studies in another UHV chamber on similarly prepared samples which were also characterized by LEED and AES. At submonolayer coverages, $\mathrm{Pt}(111)$ terraces were randomly covered by two-dimensional islands (Fig. 2a). The height measurements revealed that the islands are predominantly of $\sim 2 \AA$ in apparent height, although a few islands of $\sim 4 \AA$ in height were also observed (see line profile in Fig. $2 d$ ). These values correspond to one and two layers of graphite-like $\mathrm{ZnO}$, respectively. (Henceforth, we will refer to the amount of $\mathrm{Zn}$ which forms a dense monolayer film as one monolayer equivalent (MLE)). Both mono- and bi-layer islands showed Moiré superstructure with a 17 periodicity (Figs. $2 c$ ), which is consistent with the $\operatorname{Pt}(111)-(6 \times 6)$ superstructure observed by LEED. For the "thick" films (> 4 MLE) no more Moiré structure is observed by STM and LEED. However, triangular pits and islands (Fig. 2e), which are very similar to those observed on $\mathrm{ZnO}(0001)$ single crystal surfaces(16; 17) appear. 

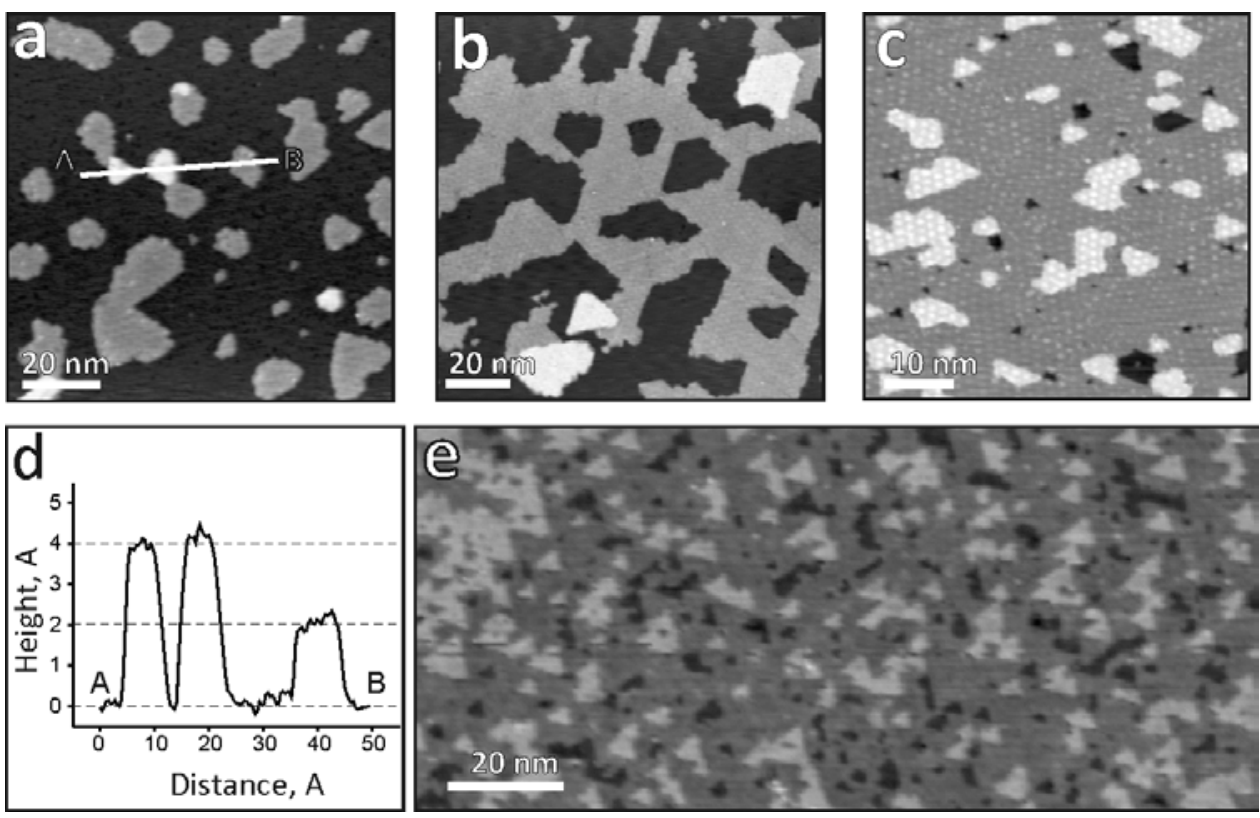

Figure 2. STM images of the "as prepared" ZnO films on Pt(111) at 0.25 (a), 0.55 (b), 1.2 (c) and 4 MLE (e) coverages. Figure (d) shows a profile scan along the A-B line marked in (a).

Taken together, the LEED, AES, STM, and TPD results show that the "as prepared" films first form $\mathrm{ZnO}(0001)$ monolayer islands which coalesce until the film covers the entire metal substrate. The films then grow in a layer-by-layer mode. Upon increasing the thickness to above $4 \mathrm{MLE}$, the film surface is essentially the same as that of a $\mathrm{ZnO}(0001)$ single crystal, although the surface termination has not yet been identified.

The $\mathrm{ZnO}$ films, characterized by LEED, AES and CO TPD, were then examined in the CO oxidation reaction at near-atmospheric pressures at $450 \mathrm{~K}$. The reactivity measurements were performed in an excess of oxygen $\left(\mathrm{CO} / \mathrm{O}_{2}=1 / 5\right)$ in order to prevent any possible oxide reduction and film dewetting, and also to compare with the previously studied $\mathrm{FeO} / \mathrm{Pt}(111)$ films, which exhibited film dewetting under oxygen-lean conditions.(18; 19)

Figure 3 shows $\mathrm{CO}_{2}$ production rate as a function of the nominal film thickness measured prior to the reaction. It is therefore clear, that the reaction exhibits a strong rate enhancement at sub-monolayer coverage. Interestingly, the 1 MLE film shows some activity, at least, it is higher than on pure $\mathrm{Pt}(111)$. As the thickness further increases, the rate gradually decreases such that the multilayer, "thick" ZnO films become almost inert under the conditions 
studied. Note also that the reaction kinetics observed for the most active $\mathrm{ZnO}$ films show no deactivation in time.

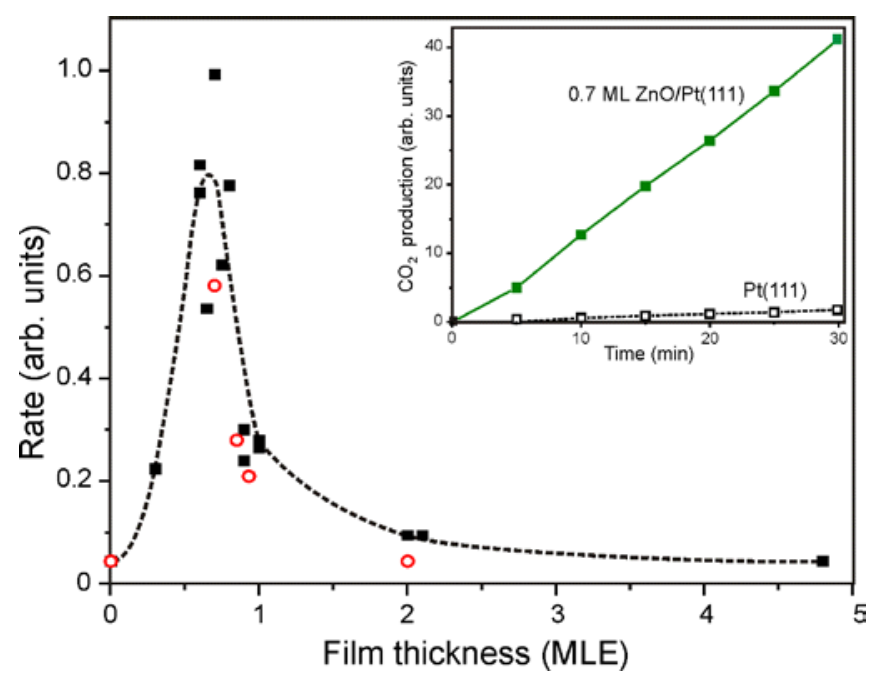

Figure 3. $\mathrm{CO}_{2}$ production rate measured over $\mathrm{ZnO}$ films on $\mathrm{Pt}(111)$ as a function of the nominal film thickness (in MLE). Solid and open symbols show data for the films prepared using reactive $\mathrm{Zn}$ deposition at 85 and $300 \mathrm{~K}$, respectively. Note that for the sub-monolayer films, the film thickness corresponds to the surface coverage as measured by $\mathrm{CO}$ titration of $\mathrm{Pt}(111)$. The inset shows kinetic curves for $\mathrm{CO}_{2}$ production on the pure $\mathrm{Pt}(111)$ and 0.7 $\mathrm{ML} \mathrm{ZnO(0001)} \mathrm{film.} \mathrm{Reaction} \mathrm{conditions:} 10 \mathrm{mbar} \mathrm{CO}+50 \mathrm{mbar}_{2}$, He balance to 1 bar; $450 \mathrm{~K}$.

AES characterization of the spent catalysts did not reveal considerable changes in the film stoichiometry, although the samples were lightly contaminated with carbon, particularly at the sub-monolayer coverages. LEED patterns showed diffuse $(1 \times 1)$ spots of $\mathrm{Pt}(111)$ and $\mathrm{ZnO}(0001)$, whereas the $(6 \times 6)$ superstructure spots vanished (Fig. 4). Subsequent TPD measurements showed a considerably higher $\mathrm{CO}$ uptake as compared to that of measured prior to the reaction, thus indicating film dewetting and concomitant Pt surface opening under the reaction conditions (Fig. S4). 

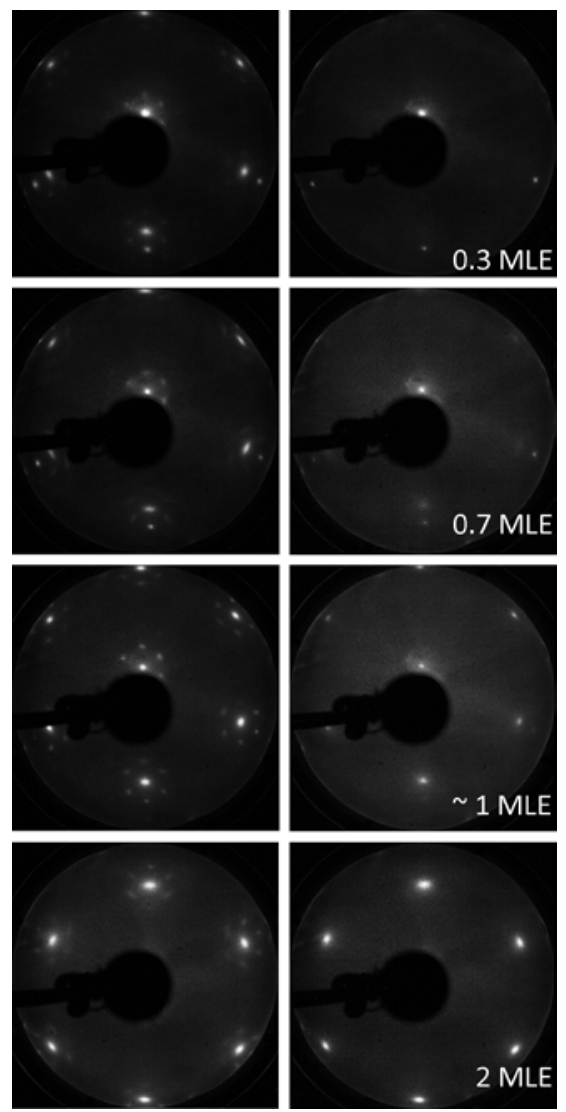

Figure 4. LEED patterns (60 eV) of the ZnO films on $\mathrm{Pt}(111)$ before (left panel) and after (right panel) the reaction. The film thickness is indicated.

The morphology of the catalysts was studied with STM ex situ. For this, the well-defined films were exposed to the same reaction conditions (10 mbar $\mathrm{CO}, 50 \mathrm{mbar}_{2}$, He balance to 1 bar, $450 \mathrm{~K}$ ) in a high-pressure cell for $10 \mathrm{~min}$. The sample was evacuated and transferred into the analytical chamber via a gate valve and immediately scanned with STM at room temperature. The STM images of the "reacted" samples are displayed in Fig. 5. For the 0.25 MLE film, irregularly shaped islands of $\sim 10 \mathrm{~nm}$ in lateral size are observed (Fig. 5a). The film coverage is reduced to $\sim 0.18 \mathrm{ML}$ (which is most likely overestimated due to the well-known tip convolution effect for small objects). Monolayer islands, which dominated the surface of the "as prepared" film (see Fig. 2a), are no longer observed. Instead, the particles are all about $4 \AA$ in height, thus indicating the formation of two-layers thick (henceforth "bilayer") $\mathrm{ZnO}(0001)$ islands. Similar behavior is observed for the 0.55 MLE film (Fig. 5b): The surface coverage 
decreases to $~ 0.32 \mathrm{ML}$, and the Pt(111) support is covered with small $\sim 4 \AA$-high islands and their aggregates. Finally, the 1.2 MLE film dewets as well, thus forming a $0.75 \mathrm{ML}$ covered "bilayer" film (Fig. 5d). For all films inspected, only small amounts of $\mathrm{ZnO}$ are found in the third layer. The long range ordering via the Moiré $(6 \times 6)$ superstructure seen on the "as prepared" films is hardly visible on the "reacted" films, in full agreement with the LEED data (Fig. 4).

The STM results show that the $\mathrm{CO}$ oxidation reaction on $\mathrm{ZnO} / \mathrm{Pt}$ is accompanied by substantial structural transformations such that monolayer islands (and entire monolayer films) transform into the bilayer islands and their aggregates. Therefore, the Pt surface opening observed by CO uptake measurements of the spent catalyst reflects a higher stability of the bilayer structure at elevated pressures. The exact mechanism and the driving force for the observed structural changes are hard to envision on the basis of the existing results. Note, however, that bilayer $\mathrm{ZnO}$ islands were also stabilized on $\mathrm{Pd}(111)$ at increasing oxygen pressures used for oxidation at $550 \mathrm{~K}$.(10) Interestingly, bilayer islands at sub-monolayer oxide coverages were also observed for $\mathrm{CeO}_{2}(111)$ films on $\mathrm{Rh}(111)(20)$ and $\mathrm{Ru}(0001)$.(21)
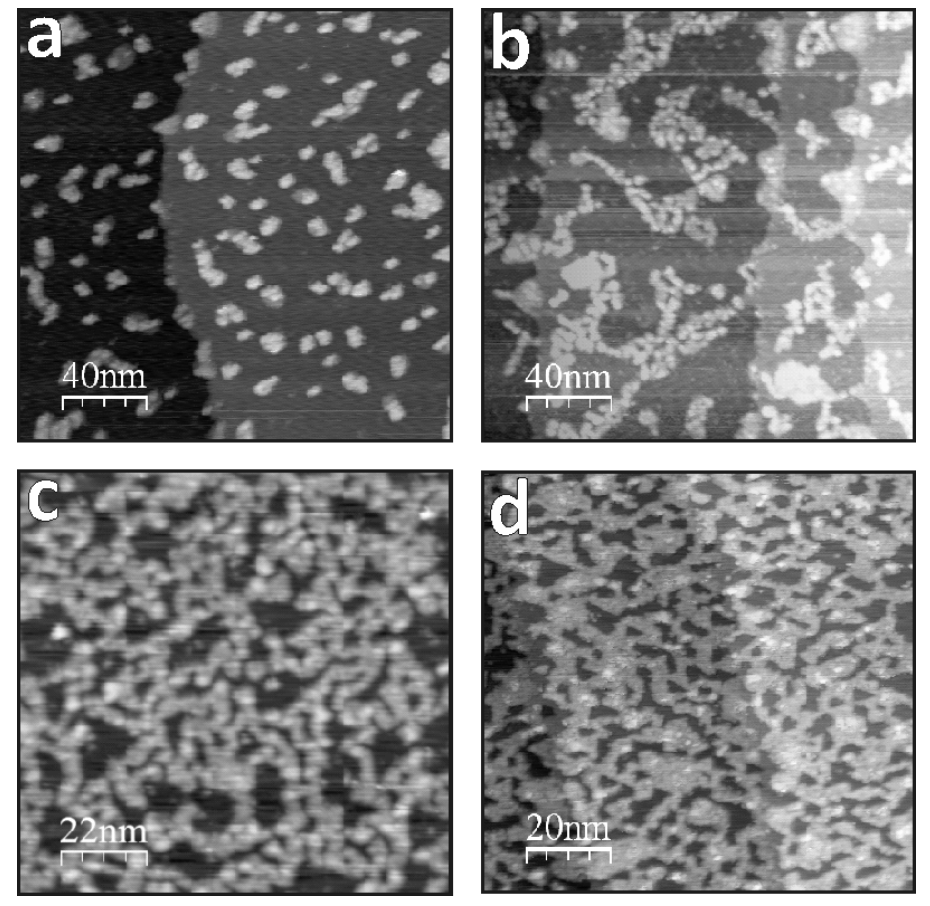

Figure 5. STM images of the "reacted" ZnO films at 0.25 (a), 0.55 (b), 0.8 (c) and 1.2 MLE (d) film thicknesses. 
The rate enhancement on the metal surfaces partially covered by oxide is commonly rationalized in terms of the reactions occurring at the oxide/metal boundary, which have been studied on the so called "inverse model catalysts" (see, for example, refs. (22-24)). Its perimeter also reaches a maximum at sub-monolayer coverage and goes to zero for a dense oxide film. In order to validate such a correlation, Figure 6 shows the perimeter of the oxide islands, measured by STM on the "reacted" samples (see Fig. 5) and normalized to the surface area, as a function of the film nominal thickness measured prior to the reaction. This plot shows a fairly good correlation with reactivity data shown in Fig. 3, although the precise position of the maximum may deviate since the STM measurements were performed ex situ in another experimental setup.

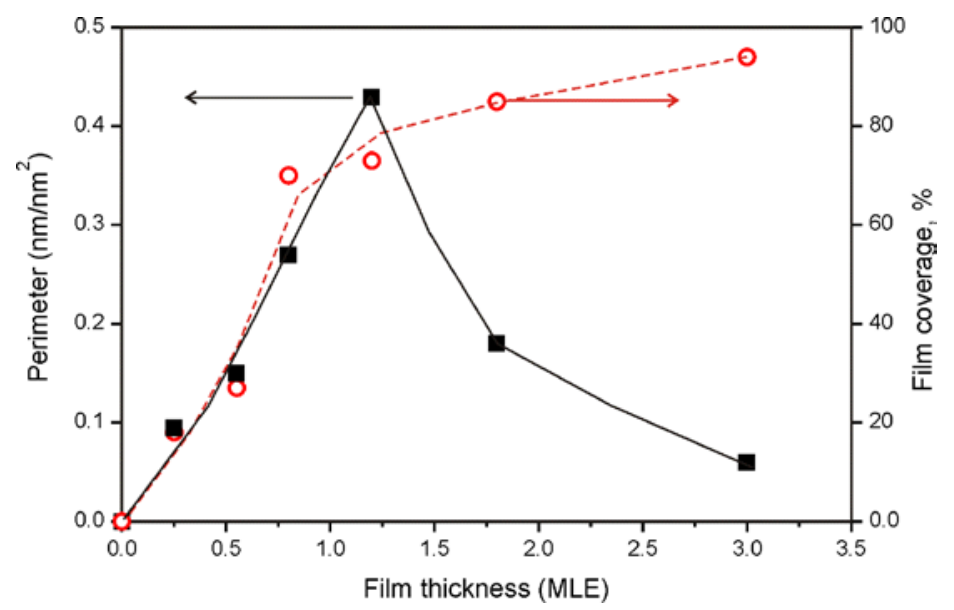

Figure 6. The perimeter of oxide/metal boundary and the film coverage measured by STM on the "reacted" samples as a function of the nominal film thickness measured on the same samples before the reaction (10 mbar $\mathrm{CO}+50 \mathrm{mbar} \mathrm{O} 2$, He balance; $450 \mathrm{~K} ; 10 \mathrm{~min})$.

In principle, the metal-oxide synergy effect may result either from oxygen spillover from oxide to Pt or from the formation of highly active sites at the metal/oxide boundary. Since ZnO is non-reducible oxide, the spillover mechanism seems to be unlikely.

Previously, a maximum in low temperature $\mathrm{CO}$ oxidation in the sub-monolayer regime was reported by Hardacre et al.(25) for the $\mathrm{CeO}_{x} / \mathrm{Pt}(111)$ system. The ceria coverage was 
measured by titration of bare Pt(111) with CO. Reactivity was measured in a high-pressure cell used as a batch reactor filled with $~ 10$ Torr of stoichiometric (2:1) $\mathrm{CO}+\mathrm{O}_{2}$ mixture at a crystal temperature of $320-430 \mathrm{~K}$. The authors found that the reaction rate increased between zero and 0.5 ML, after which it dropped almost to zero at 0.8-1.3 ML, but again increased steeply to a value which is much greater than that observed over the clean Pt(111) surface. It was suggested that for coverages below $1 \mathrm{ML}$ the reactivity is associated with the metal/oxide interface. The high reactivity of the thick ceria films, fully encapsulating Pt(111) (as judged by CO TPD before the reaction), was rationalized in terms of a proposal made by Frost,(26) according to which electron transfer from a metal phase to an oxide phase reduces the enthalpy for oxygen vacancy formation in the oxide. However, the authors themselves argued that there was no absolute evidence of total encapsulation of the Pt crystal. In particular, the sample morphology after the reaction was undefined in this work. In principle, the initially dense oxide film could dewet under reaction conditions, ultimately forming ceria particles on $\operatorname{Pt}(111)$.

Nonetheless, the rate vs. thickness plot observed for the $\mathrm{CeO}_{2-x}(111) / \mathrm{Pt}(111)$ system is very different from that shown in Fig. 2 for $\mathrm{ZnO}(0001) / \mathrm{Pt}(111)$. While "thick" ceria films ( 10 MLE) were much more active than $\mathrm{Pt}(111)$, the reactivity of $\mathrm{ZnO}(0001) / \mathrm{Pt}(111)$ dies away with increasing the film thickness. The difference may be related to the fact that ceria, well-known for its oxygen storage-release properties, readily provides weakly bound oxygen to react with $\mathrm{CO}$, whereas ZnO does not.

Recently Sun et al.(27) performed density functional theory studies of the $3 \mathrm{~d}$ transitionmetal oxide (TMO) nano-islands on Pt(111) in low-temperature CO oxidation in order to identify the active sites at the TMO/Pt boundaries. A Pt-cation ensemble was proposed, where coordinatively unsaturated TMO cations exposed at the edges of oxide nanoislands are highly active for $\mathrm{O}_{2}$ adsorption and dissociation, and less-reactive $\mathrm{Pt}$ binds modestly with dissociated $\mathrm{O}$ responsible for the facile CO oxidation. However, in all these calculations the TMO islands were only one monolayer in thickness, which is definitely not the case under technologically relevant conditions as shown here for $\mathrm{ZnO}(0001)$ and previously for $\mathrm{FeO}(111)$ on $\mathrm{Pt}(111)$.(3) Therefore, our results provide more adequate structural models for TMO/metal "inverted" catalysts. 
It is noteworthy that interfacial sites at oxide-metal interfaces have been long considered as active sites responsible for reactivity of oxide-supported metal catalysts. However, the proof has been exclusively based on density functional calculations of simplified models. Also, there were no experimental studies reported on model systems which could be directly correlated with the theoretical models. One of the very few studies where a direct comparison between calculations and experimental model studies has been carried out is for the above mentioned FeO/Pt system. Very recently, Schlögl and his group have shown that a $\mathrm{Pd}$ /iron oxide powder catalyst also exhibits encapsulation of metal particles by an iron oxide overlayer that enhances reactivity.(28) In this case the metal is completely covered with FeO, and therefore there is no accessible oxide/metal interface to hold responsible for the increased reactivity. Control model system experiments with a fully covered film as opposed to a partially dewetted film have been carried out to confirm this statement.

In the present case the corresponding experiments on fully covered $\mathrm{ZnO}$ films as well as on partially dewetted films have been performed, and here the situation is opposite to the case of FeO/Pt. There are no directly comparable experiments available in the literature. Note, however, that Behrens et al. have recently presented evidence on $\mathrm{ZnO} / \mathrm{Cu}$ methanol synthesis powder catalysts together with density functional calculations that it is the $\mathrm{ZnO} / \mathrm{Cu}$ interface that is responsible for the high activity.(29) Again, there are no directly comparable experimental model studies available to validate or disprove this conclusion. The present data suggest that, indeed, the $\mathrm{ZnO} /$ metal interface may be responsible for reactivity of $\mathrm{ZnO}$ based catalysts, although a direct verification is yet to be seen.

\section{Conclusions}

Well-ordered $\mathrm{ZnO}(0001)$ films on $\mathrm{Pt}(111)$ were studied in the $\mathrm{CO}$ oxidation reaction as a function of the film thickness and coverage. At low temperatures (450 K) and near-atmospheric pressures, $\mathrm{CO}_{2}$ production is found to be much higher on the partially covered films than on the dense $\mathrm{ZnO}(0001)$ films and bare $\mathrm{Pt}(111)$. Under reaction conditions, monolayer structures transform into bilayer-thick islands, which dominate the surface of the active catalysts. The 
observed structure-reactivity relationships directly show that the oxide/metal boundary provides the active sites. The results provide a more adequate structural model than previously suggested for elucidating the reaction mechanism on the oxide/metal boundary at technologically relevant conditions.

\section{Acknowledgments}

BHL acknowledges the International Max Planck Research School "Complex surfaces in materials science". IMNG thanks the Alexander von Humboldt Foundation for a fellowship. MEM was a Fulbright Grantee in the Graduate Fellow Student Program 2011-2012. The work has been supported by Fonds der Chemischen Industrie, Deutsche Forschungsgemeinschaft, and Cluster of Excellence "UniCat". 


\section{References}

[1] S. Shaikhutdinov, H.-J. Freund, Annual Review of Physical Chemistry 63 (2012) 619-633.

[2] H.-J. Freund, G. Pacchioni, Chemical Society Reviews 37 (2008) 2224-2242.

[3] Y.-N. Sun, L. Giordano, J. Goniakowski, M. Lewandowski, Z.-H. Qin, C. Noguera, S. Shaikhutdinov, G. Pacchioni, H.-J. Freund, Angewandte Chemie-International Edition 49 (2010) 4418-4421.

[4] M. Lewandowski, Y.N. Sun, Z.H. Qin, S. Shaikhutdinov, H.J. Freund, Applied Catalysis a-General 391 (2011) 407-410.

[5] , Journal of the American Chemical Society 131 (2009) 16636-

16637.

[6] F.F. Vol'kenshtein, Russian Chemical Reviews 35 (1966) 537.

[7] G.-M. Schwab, in: H.P. D.D. Eley, B.W. Paul (Eds.), Advances in Catalysis, 1979, pp. 1-22.

[8] A.A. Slinkin, E.A. Fedorovskaya, Russian Chemical Reviews 40 (1971) 860.

[9] C. Tusche, H.L. Meyerheim, J. Kirschner, Physical Review Letters 99 (2007) 026102.

[10] G. Weirum, G. Barcaro, A. Fortunelli, F. Weber, R. Schennach, S. Surnev, F.P. Netzer, The Journal of Physical Chemistry C 114 (2010) 15432-15439.

[11] C. Wöll, Progress in Surface Science 82 (2007) 55-120.

[12] E. Weiss, M. Folman, Journal of the Chemical Society, Faraday Transactions 1: Physical Chemistry in Condensed Phases 82 (1986) 2025-2041.

[13] W. Doerffler, K. Hauffe, Journal of Catalysis 3 (1964) 171-178.

[14] M. Kobayashi, B. Golman, T. Kanno, Journal of the Chemical Society, Faraday Transactions 91 (1995) 1391-1398.

[15] P. Esser, R. Feierabend, W. Göpel, Berichte der Bunsengesellschaft für physikalische Chemie 85 (1981) 447-455.

[16] O. Dulub, L.A. Boatner, U. Diebold, Surface Science 519 (2002) 201-217.

[17] O. Dulub, U. Diebold, G. Kresse, Physical Review Letters 90 (2003) 016102.

[18] Y.N. Sun, Z.H. Qin, M. Lewandowski, E. Carrasco, M. Sterrer, S. Shaikhutdinov, H.J. Freund, Journal of Catalysis 266 (2009) 359-368.

[19] Y.-N. Sun, Z.-H. Qin, M. Lewandowski, S. Kaya, S. Shaikhutdinov, H.-J. Freund, Catalysis Letters 126 (2008) 31-35.

[20] S. Eck, C. Castellarin-Cudia, S. Surnev, M.G. Ramsey, F.P. Netzer, Surface Science 520 (2002) 173185.

[21] J.L. Lu, H.J. Gao, S. Shaikhutdinov, H.J. Freund, Surface Science 600 (2006) 5004-5010.

[22] J. Schoiswohl, S. Eck, M.G. Ramsey, J.N. Andersen, S. Surnev, F.P. Netzer, Surface Science 580 (2005) 122-136.

[23] K. Hayek, B. Jenewein, B. Klötzer, W. Reichl, Topics in Catalysis 14 (2000) 25-33.

[24] A.B. Boffa, C. Lin, A.T. Bell, G.A. Somorjai, Catalysis Letters 27 (1994) 243-249.

[25] C. Hardacre, R.M. Ormerod, R.M. Lambert, The Journal of Physical Chemistry 98 (1994) 1090110905.

[26] J.C. Frost, Nature 334 (1988) 577-580.

[27] D. Sun, X.-K. Gu, R. Ouyang, H.-Y. Su, Q. Fu, X. Bao, W.-X. Li, The Journal of Physical Chemistry C 116 (2012) 7491-7498.

[28] H.-J. Freund, G. Meijer, M. Scheffler, R. Schlögl, M. Wolf, Angewandte Chemie International Edition 50 (2011) 10064-10094.

[29] M. Behrens, et al., Science 336 (2012) 893-897. 